\title{
Ensino de Informática: Habilidades Requeridas para a Sociedade 5.0
}

\author{
Gabriel Moreira', Ricardo Silva², Giorgia Mattos ${ }^{1}$, Josilene Aires ${ }^{1}$ \\ ${ }^{1}$ Universidade Federal da Paraíba, Centro de Informática \\ ${ }^{2}$ Universidade Federal da Paraíba, Centro de Tecnologia \\ moreiragabriel.aires@gmail.com, ricardomoreira0203@hotmail.com, \\ \{giorgia,josilene\} @ci.ufpb.br
}

\begin{abstract}
Technological advances transform the way that work tasks are divided between humans and machines, strongly impacting the labor market. Computer science education needs to prepare students for Society 5.0, encouraging them to learn new skills so that they are key players in this transition. This article presents the characteristics of this society and lists the main skills and technologies for the professional life. The clusters with greatest opportunities are: (i) Artificial Intelligence (AI) and Data Science and (ii) Engineering and Cloud Computing; the essential technological axes are: Internet of Things, AI, Robotics and Distributed Record Technologies.
\end{abstract}

Resumo. Os avanços tecnológicos transformam a maneira que as tarefas de trabalho são divididas entre os seres humanos e as máquinas, impactando fortemente o mercado de trabalho. O ensino de Informática necessita preparar os estudantes para a Sociedade 5.0, estimulando-os a novos aprendizados para que sejam atores-chave nesta transição. Este artigo apresenta as características desta sociedade e elenca as principais habilidades e tecnologias essenciais para o profissional. Os clusters profissionais com maiores oportunidades são: (i) Inteligência Artificial (IA) e Ciência de Dados e (ii) Engenharia e Cloud Computing; já os eixos tecnológicos essenciais são: Internet das coisas, IA, Robótica e Tecnologias de Registros Distribuídos.

\section{Introdução}

Uma série de revoluções do passado, incluindo as revoluções agrícolas e industriais, trouxeram não apenas avanços tecnológicos, mas também mudanças estruturais para a sociedade. A sociedade tem evoluído desde a Sociedade 1.0 (Caça e Coleta), passando pela Sociedade Agrária (2.0), pela Sociedade Industrial (3.0) até a Sociedade da Informação (4.0); a partir de 2016, o Japão anunciou o surgimento da Sociedade 5.0 [Keidanren 2018]. Em cada uma dessas sociedades, diferentes ferramentas e habilidades eram requeridas, conforme a Figura 1.

Como podemos perceber, os seres humanos passaram por três sociedade sem o uso de computadores. Suas habilidades e cognições tratavam muito mais da sobrevivência física e da segurança do que da preocupação com qualidade de vida, onde o lazer e a autorrealização estariam presentes. Embora seja difícil prever qual futuro a chamada "Sociedade 5.0" nos reserva, podemos nos antecipar e atuar como atores-chave neste processo de mudança, compreendendo a evolução da sociedade e procurando nos antecipar às necessidades emergentes no campo do trabalho. 


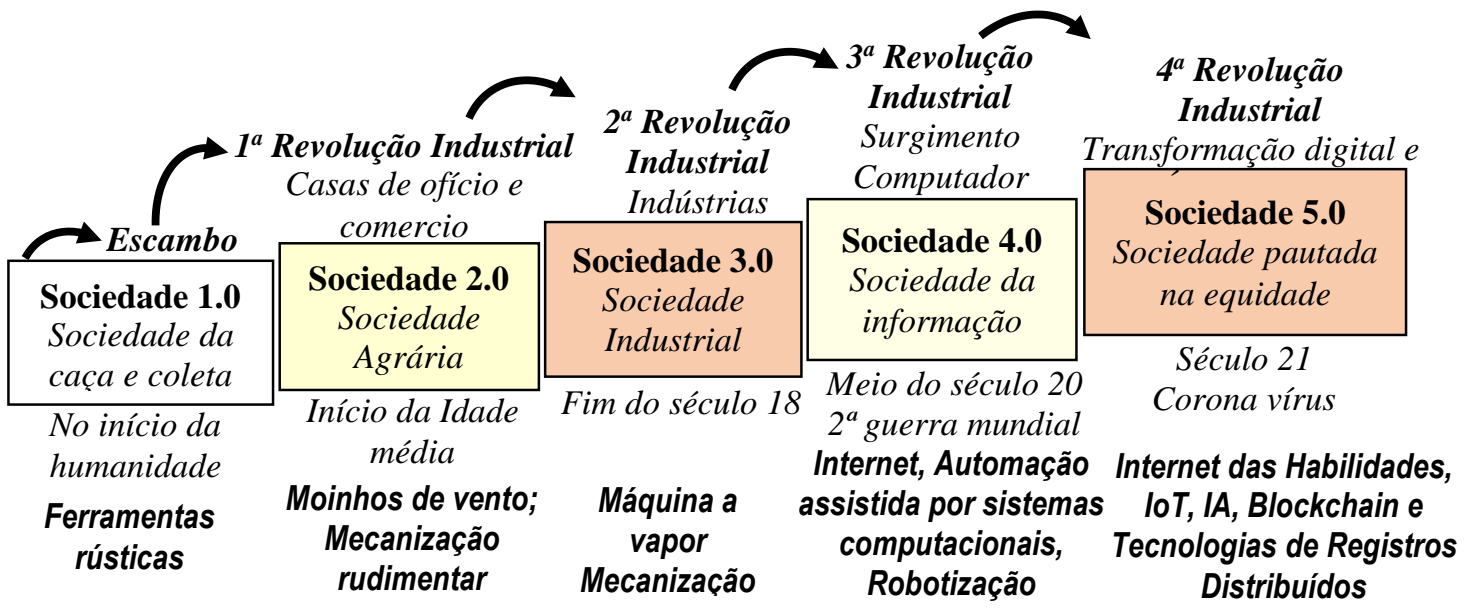

Figura 1: Evolução da Sociedade X Revoluções Industriais X Ferramentas

Esta pesquisa utiliza os métodos de bibliometria e netnografia. Enquanto a bibliometria baseia-se em artigos científicos, a netnografia amplia as possibilidades oferecidas pela etnografia, ao permitir o estudo de objetos, fenômenos e culturas que emergem no ciberespaço [Corrêa e Rozados 2017]. Surgiu em 1995 (netnografia nethnography $=$ net + ethnography), com um desafio: preservar os detalhes ricos da observação em campo etnográfico usando o meio eletrônico [Correia et al. 2017; Kozinets 2014]. A seguir apresentaremos a conceituação de Sociedade 5.0 e suas características; a seção 3 apresenta as tecnologias envolvidas na sua consecução; a seção 4 apresenta as oportunidades e habilidades profissionais nesta Sociedade e, por fim, as conclusões na seção 5 .

\section{A Sociedade 5.0}

A iniciativa intitulada Sociedade 5.0 (Society 5.0) foi proposta em 2016 através do Quinto Plano Básico de Ciência e Tecnologia do Japão, com a visão de criar uma "sociedade super inteligente" (Super Smart Society) onde os seres humanos terão como paradigma principal a equidade de valores econômicos, sociais, vivendo de forma igual, em um meio ambiente favorável à vida [Japan 2016]. Seu principal objetivo é criar uma sustentabilidade centrada no ser humano, melhorando a qualidade de vida das pessoas através de sistemas cibernéticos [Shiroishi et al. 2018]. As tecnologias são apenas o meio pelo qual os serviços deverão ser providos: é fundamental que o ser humano permaneça como o ator central, a fim de trazer felicidade e senso de valor às pessoas.

Suas características principais são, de acordo com Keidanren (2018): (a) Satisfação das necessidades individuais e criação de valor. A produção em larga escala, eficiência e alto consumo será reduzido; os bens e serviços vão deixar de ser padronizados, visando a satisfação individual. (b) Diversidade. Pessoas diferentes serão capazes de viver e trabalhar sem discriminações de gênero, raça e nacionalidade. (c) Descentralização. Busca da igualdade e distribuição de informação e saúde para todos, seja em que lugar estiverem. (d) Resiliência. As pessoas terão o equilíbrio mental pois deverão ser liberados da ansiedade causada pela falta de segurança, pelo terrorismo, pelos ataques cibernéticos e pelas catástrofes naturais como terremotos e inundações.

A infraestrutura irá melhorar a capacidade de lidar com problemas, adaptar-se a mudanças e superar obstáculos. O desemprego e a pobreza irão diminuir e (e) 
Sustentabilidade. Na Sociedade 4.0 havia consumo massivo de recursos com alto impacto ambiental, na Sociedade 5.0 busca-se a harmonia com a natureza, com abastecimento de água e gestão de resíduos sustentáveis, aumento da eficiência energética sem dependência dos recursos fósseis.

$\mathrm{Na}$ percepção do relatório elaborado por Keidanren (2018) a tecnologia computacional de ponta é que possibilitará a verdadeira Sociedade 5.0, quando tratará da criação de valor a qualquer hora, em qualquer lugar, com segurança e em harmonia com a natureza, alinhado com as 17 metas de desenvolvimento sustentável da ONU [ONU 2020; STI 2017].

\section{Tecnologias Envolvidas na Construção da Sociedade 5.0}

A transformação digital necessária para alavancar a criação da nova Sociedade 5.0 envolve o desenvolvimento e a implementação massiva de quatro áreas bem definidas da tecnologia: (i) Internet das Coisas (IoT), (ii) Inteligência Artificial, (iii) Robótica, e (iv) Blockchain e Tecnologias de Registros Distribuídos [Japan 2018; STI 2017; Kitano 2017; Warburg 2016; Ølnes et al. 2017]:

(a) Internet das Coisas. O conceito de Internet das Coisas compreende a visão de que os objetos do dia a dia estejam conectados à Internet. Para que esta realidade seja concretizada, são necessárias tecnologias que capturem dados em tempo real a partir do mundo à nossa volta e os transmitam ao ciberespaço. Na situação idealizada pelo projeto da Sociedade 5.0, o desafio é ser possível converter qualquer tipo de atividade do mundo real em dados suscetíveis a serem processados no mundo digital. Neste contexto, é necessário abranger todos e quaisquer eventos do mundo real.

(b) Inteligência Artificial As capacidades da IA foram melhoradas pelo surgimento do deep learning e por uma série de avanços tecnológicos no campo de aprendizado de máquina, bem como ambientes nas Ciência de Dados e Big Data. O maior potencial da IA encontra-se na capacidade de trabalhar com o reconhecimento e a previsão de comportamentos para sistemas complexos e a alta precisão de execução de operações, proporcionando as informações essenciais para a tomada de decisão em problemas complexos de serem analisados por outras tecnologias. Quando os sistemas de IA adquirem um alto nível de habilidade, os usuários podem compartilhar os sistemas e desfrutar de habilidades qualificadas sem depender de suas habilidades individuais.

(c) Robótica. O uso da robótica combinada com a IA proporcionará o crescimento da sua utilização na manufatura e nos serviços. O desenvolvimento destas tecnologias associadas permitirá que os robôs desempenhem papéis nos lares, escritórios e áreas urbanas, além de substituir as pessoas em tarefas perigosas e repetitivas do dia a dia. Exemplo: Quando a IA é incorporada ao veículo autônomo (que é considerado um tipo de robô), este adquire habilidades que tornam a sociedade mais "inteligente".

(d) Blockchain e Tecnologias de Registros Distribuídos. Bitcoin, lançado em jan/2009, foi o primeiro sistema a incorporar a tecnologia de registros distribuídos (blockchain) em suas transações, tendo introduzido confiabilidade em um ambiente intrinsicamente inseguro como a web. Baseia-se em criptografia de chaves públicas e assinaturas digitais em um ambiente distribuído de redes P2P. Provê alta integridade de dados em aspectos como consistência, segurança, e confiabilidade, dificultando a manipulação de dados e a introdução de informações falsas nos sistemas que as utilizam. 
Este conjunto de tecnologias possibilitarão a chamada "Internet das Habilidades", onde as capacidades humanas e da IA poderão ser transferíveis, interconectáveis e funcionar de forma sinérgicas. Poderá haver impacto negativo, reduzindo empregos; entretanto, esse problema é antigo, observado desde a década 50 quando a tecnologia começou a avançar. É aconselhável aos profissionais de Informática para procurem adquirir as habilidades relativas a estas tecnologias, a fim de moldarem-se às novas demandas, evitando o desemprego [Rekimoto 2016].

\section{Habilidades Profissionais na Sociedade 5.0}

A transformação digital requerida pela Sociedade 5.0 será possível através do desenvolvimento das habilidades dos profissionais das áreas de tecnologia, a fim de tornar possível a sua implantação. Os dados descritos aqui resumem pesquisas que mostram as tendências reais do mercado de trabalho mundial, a partir de dados das empresas LinkedIn, da Burning Glass Technologies e do Fórum Econômico Mundial, utilizando a metodologia de netnografia, conforme descrito anteriormente. Estas entidades mapeiam as oportunidades existentes e projetam as habilidades necessárias para a nova economia que está surgindo, apresentando-as em forma de pesquisas e relatórios. Para o nosso mapeamento, tomaremos como base três destes relatórios:

R1: "Jobs of Tomorrow - Mapping Opportunity in the New Economy". Utiliza fontes que informam as oportunidades de trabalho nos últimos cinco anos para as vagas e habilidades futuras [Ratcheva et al. 2020].

R2: "Global Al Talent Trends: Looking back at Al's impact in 2019 to understand the challenges and opportunities ahead". Examina oportunidades futuras de trabalho em IA [Berger 2019].

R3: "What's Trending in Jobs and Skills". Foram examinadas 95 milhões de vagas de emprego entre 2015 e 2018, projetando-as para o futuro [Strack et al. 2019].

As profissões foram analisadas e agrupadas em clusters que apresentaram a maior demanda profissional entre 2015 e 2019, para então delinear a demanda futura. Vamos enfatizar aqui a área de TI, na qual destacam-se dois clusters profissionais: (i) Ciência de Dados e IA e (ii) Engenharia e Computação em Nuvem (Cloud Computing). A Tabela 1 mostra as oportunidades profissionais emergentes (Demanda Profissional - a) e as habilidades exigidas (Habilidades - b) nestes dois clusters.

As oportunidades profissionais emergentes estão também classificadas em (*) pequena-escala e (**) larga-escala, indicando a demanda que será necessária para cada profissão. Quanto às habilidades necessárias em cada cluster profissional (Habilidade), foram diferenciadas as habilidades em duas categorias, (\#) tecnologia disruptiva e (\#\#) tecnologia básica. 
Tabela 1. Demandas profissionais e habilidades chave para a Sociedade 5.0.

Fonte: Adaptado dos Relatórios 1, 2 e 3

\begin{tabular}{|c|c|c|c|}
\hline \multicolumn{2}{|c|}{ Demanda Profissional (a) } & \multicolumn{2}{|c|}{ Habilidades (b) } \\
\hline $\begin{array}{l}\text { Ciência de Dados e } \\
\text { Inteligência Artificial }\end{array}$ & $\begin{array}{c}\text { Engenharia e } \\
\text { Computação em } \\
\text { Nuvem }\end{array}$ & $\begin{array}{l}\text { Ciência de Dados e } \\
\text { Inteligência Artificial }\end{array}$ & $\begin{array}{c}\text { Engenharia e } \\
\text { Computação em } \\
\text { Nuvem }\end{array}$ \\
\hline $\begin{array}{l}1 \text { Especialista em } \\
\text { Inteligência Artificial }\left(^{*}\right)\end{array}$ & $\begin{array}{l}1 \text { Engenheiro de } \\
\text { segurança em Cloud }\left(^{*}\right)\end{array}$ & 1 Ciência de dados (\#) & 1 Desenvolvedor (\#) \\
\hline 2 Cientista de Dados $\left(^{\star}\right)$ & $\begin{array}{l}2 \text { Engenheiro } \\
\text { desenvolvedor Python } \\
\left({ }^{*}\right)\end{array}$ & $\begin{array}{l}2 \text { Tecnologias de } \\
\text { armazenamento de } \\
\text { dados (\#\#) }\end{array}$ & $\begin{array}{l}2 \text { Desenvolvedor Web } \\
(\# \#)\end{array}$ \\
\hline $\begin{array}{l}3 \text { Engenheiro de Dados } \\
\left({ }^{\star}\right)\end{array}$ & $\begin{array}{l}3 \text { Engenheiro } \\
\text { desenvolvedor Full Stack } \\
\left({ }^{* *}\right)\end{array}$ & $\begin{array}{l}3 \text { Ferramentas de } \\
\text { desenvolvimento (\#) }\end{array}$ & $\begin{array}{l}3 \text { Tecnologias de } \\
\text { armazenamento de } \\
\text { dados (\#\#) }\end{array}$ \\
\hline $\begin{array}{l}4 \text { Desenvolvedor de } \\
\text { Dados }\left({ }^{\star \star}\right)\end{array}$ & $\begin{array}{l}4 \text { Engenheiro } \\
\text { Desenvolvedor Java } \\
\text { Script }\left(^{* *}\right)\end{array}$ & 4 Inteligência artificial (\#) & $\begin{array}{l}4 \text { Ciclo de vida de } \\
\text { desenvolvimento de } \\
\text { software (SDLC) (\#\#) }\end{array}$ \\
\hline 5 Analista de Dados & $\begin{array}{l}5 \text { Engenheiro } \\
\text { Desenvolvedor Back } \\
\text { End }\left(^{* *}\right)\end{array}$ & $\begin{array}{l}5 \text { Ciclo de vida de } \\
\text { desenvolvimento de } \\
\text { software (SDLC) (\#\#) }\end{array}$ & $\begin{array}{l}5 \text { Redes de } \\
\text { computadores }(\# \#)\end{array}$ \\
\hline \multicolumn{2}{|c|}{$\begin{array}{l}\text { Escala de oportunidades } \\
\qquad \begin{array}{c}\left(^{*}\right) \text { pequena-escala } \\
\left({ }^{\star *}\right) \text { larga-escala }\end{array}\end{array}$} & \multicolumn{2}{|c|}{$\begin{array}{l}\text { Tipo de habilidade } \\
\text { (\#) tecnologia disruptiva } \\
\text { (\#\#) tecnologia básica }\end{array}$} \\
\hline
\end{tabular}

A demanda profissional é apresentada por cluster, significando por exemplo que os profissionais com maiores oportunidades nas áreas de Ciência de Dados e Inteligência Artificial são, nesta ordem: (1) Especialista em Inteligência Artificial, (2) Cientista de Dados, (3) Engenheiro de Dados, e assim por diante. Exemplificando a parte de Habilidades, temos que na área de Engenharia e Computação em Nuvem as habilidades mais requeridas serão (1) Desenvolvedor, (2) Desenvolvedor Web, (3) Tecnologias de Armazenamento de Dados e assim por diante.

As tecnologias disruptivas (compreendidas como aquelas mais modernas, "fora do padrão" e por isso mais difíceis de serem aprendidas) são mais requeridas na área de Ciência de Dados e IA. Um exemplo é a própria Ciência de Dados, que é relativamente nova e com poucos profissionais; já Redes de computadores é considerada uma tecnologia básica, pois conta com maior número de profissionais especializados.

É essencial que os cursos da área de Computação incluam o desenvolvimento destas áreas nos currículos, a fim de preparar os aprendentes para esta sociedade que já se iniciou. No Brasil um número reduzido de universidades públicas federais iniciou a oferta de cursos de graduação com ênfase específica em Inteligência Artificial entre 2019 e 2020, incluindo a Universidade Federal de Goiás e a Universidade Federal da Paraíba. Sugere-se que os atuais cursos de Informática e Computação incluam em seus currículos 
o desenvolvimento das habilidades enfatizadas em nosso estudo, a fim de lançar no mercado profissionais melhor preparados para os desafios do mercado de trabalho.

\section{Conclusões}

A Sociedade 5.0 propõe o uso de tecnologias avançadas para que o homem possa obter maior equilíbrio entre a vida pessoal e profissional, ter maior senso de realização e viver de maneira sustentável, respeitando a natureza e a diversidade humana. As tecnologias mais importantes para que se alcance esta sociedade são IoT, IA, Robótica, Blockchain e DLT, de uma maneira mais ampla e integrada do que existe hoje em dia.

As profissões da área de Informática com maior demanda para este futuro que já se iniciou podem ser agrupadas em duas áreas: (i) Ciência de Dados e IA e (ii) Engenharia e Computação em Nuvem. Dentro destas áreas identificamos as oportunidades emergentes de trabalho, descrevendo tanto as profissões com maior demanda como as habilidades requeridas. Sendo assim, é essencial que os cursos de Informática adequem seus perfis para que possam preparar os aprendentes para atenderem às novas necessidades da Sociedade 5.0, tornando-os agente de mudança e alcançando sucesso em um futuro que já começou.

\section{Referências}

Berger, Guy, (2019) "Global AI Talent Trends: Looking back at AI's impact in 2019 to understand the challenges and opportunities ahead", LinkedIn Corporation. https://economicgraph.linkedin.com/blog/global-ai-talent-trends-looking-back-at-aiimpact-in-2019-to-understand-the-challenges-and-opportunities-ahead.

Corrêa, M. V. Rozados, Helen B. F. (2017) "The netnography as research method in Information Science", In: Revista eletrônica de biblioteconomia e ciência da informação, v. 22, n.49, p. 1-18, maio/ago., ISSN 1518-2924. DOI: 10.5007/15182924.2017. v22, n. 49, p 1.

Japan Government (2016). "The 5th Science and Technology Basic Plan", Government of Japan, http://www8.cao.go.jp/cstp /english/basic/5thbasicplan.pdf, January.

Keidanren. (2018). "Society 5.0 - Co-creating the Future". In: Report in Policy \& Action. www.keidanren.or.jp/en/policy/2018/095_outline.pdf.

Kitano, H. (2017), "The future of blockchains lies in linkage to artificial intelligence", In: Diamond Harvard Business Review, August.

Kozinets, Robert V. (2014) "Netnografia: Realizando pesquisa etnográfica online”, Porto Alegre: Penso.

Liu, J., Zhu, Y., Serapio, M., \& Cavusgil, S. T. (2019) "The new generation of millennial entrepreneurs: A review and call for research", In: International Business Review, 28(5), 101581. https://doi.org/10.1016/j.ibusrev.

Ølnes, Svein \& Ubacht, Jolien \& Janssen, Marijn. (2017). "Blockchain in government: Benefits and implications of distributed ledger technology for information sharing", Government Information Quarterly.

ONU, (2020) "Sustainable Development Goals: 17 Goals to Transform Our World", United Nations; http://www.un.org/sustainabledevelopment. 
Ratcheva, V., Leopold, T. A., \& Zahid, S. (2020) "Jobs of tomorrow: mapping opportunity in the new economy". World Economic Forum, Geneva, Switzerland.

Rekimoto, J. (2016), "From IoT to IoA, a network to extend humankind", Nikkei Electronics, January.

Shiroishi, Yoshihiro, Uchiyama Kunio, and Suzuki Norihiro. (2018) "Society 5.0: For Human Security and Well-Being", Hitachi Research and Development Group, IEEE.

STI. (2017) "Recommendation for the Future - STI as a Bridging Force to Provide Solutions for Global Issues: Four Actions of Science and Technology Diplomacy to Implement the SDGs", Advisory Board for the Promotion of Science and Technology Diplomacy, http://www.mofa.go.jp /files/000255801.pdf, May.

Strack, R., Kaufman, E., Kotsis, A., Sigelman, M., Restuccia, D. and Taska, B. (2019) "What's Trending in Jobs and Skills", Burning Glass, Boston, USA.

Warburg, B. (2016) "How the blockchain will radically transform the economy", https://www.ted.com/talks/bettina_warburg_how_the_blockchain_will_radically_tra nsform_the_economy?language $=\bar{e}$, June. 